УДК

КАУШИК Р., ХАНДЕЛВАЛ В., ДЖЕЙН Р. С.

\title{
ВЛИЯНИЕ УСРЕДНЕНИЯ АПЕРТУРЫ И ПРОСТРАНСТВЕННОГО РАЗНЕСЕНИЯ ПРИЕМА НА ЕМКОСТЬ КАНАЛА В ОПТИЧЕСКИХ БЕСПРОВОДНЫХ ТЕЛЕКОММУНИКАЦИОННЫХ СИСТЕМАХ С ЛОГАРИФМИЧЕСКИ НОРМАЛЬНЫМ КАНАЛОМ
}

\author{
Институт информационных технологий им. Джэйпи, \\ Индия, Ноида, Уттар-Прадеш
}

\begin{abstract}
Аннотация. В статье рассмотрена работа беспроводной телекоммуникационной системы с логарифмически нормальным каналом при использовании усреднения апертуры и разнесенного приема. Выполнена оценка средней пропускной способности (емкость канала) такой системы с помощью простых выражений с хорошей аппроксимацией. Исследуется и сравнивается качественное улучшение емкости канала при использовании технологий уменьшения турбулентности (усреднение апертуры, технология разнесенного приема) и разнесенного приема (суммирование дифференциально-взвешенных сигналов каждого канала и линейное суммирование сигналов равной мощности). Установлено, что усреднение апертуры дает существенное улучшение производительности по сравнению с обоими типами разнесенного приема вне зависимости от конкретного значения силы турбулентности. Независимо от силы турбулентности главное улучшение емкости канала достигается применением массива (сети) направленных детекторов приемника. Полученные выражения согласуются с результатами, полученными с помощью моделирования методом Монте-Карло.
\end{abstract}

Ключевые слова: оптическая беспроводная связь; средняя емкость канала; усреднение апертуры; разнесенный прием; логарифмически нормальное распределение

\section{ВВЕДЕНИЕ}

С ростом требований к пропускной способности каналов становится ясно, что используемые в настоящее время радиочастотные технологии доступа, такие как проводные технологии на медных/коаксиальных кабелях и сотовые микроволновые технологии, не могут удовлетворить возрастающие требования к емкости канала. Такие приложения как видеотелефония, мобильное телевидение и видеоигры требуют большой пропускной способности каналов, что в свою очередь требует альтернативных решений для организации доступа в таких сетях.
Сети, построенные на оптоволоконных линиях, уже стали популярными, но установка таких систем достаточно дорога и трудозатратна. Оптические беспроводные сети OWC (optical wireless communication) или оптика свободного пространства FSO (free space optics) рассматриваются как перспективные решения для удовлетворения всевозрастающих требований к увеличению скорости передачи данных и для преодоления «бутылочного горлышка» последней мили в сетях доступа с разнообразными приложениями (линии связи на короткие расстояния и линии связи «последней мили»). Они также включают в себя внешние линии связи между зданиями и кос- 


\section{БИБЛИОГРАФИЧЕСКИЙ СПИСОК}

1. Khalighi M. A. Survey on free space optical communication: A communication theory perspective / Mohammad A. Khalighi, Murat Uysal // IEEE Commun. Surveys Tutorials. - 2014. - Vol. 16, No. 4. - P. 2231-2258. - DOI : 10.1109/COMST.2014.2329501.

2. Zhu $X$. Free-space optical communication through atmospheric turbulence channels / Xiaoming Zhu, J. M. Kahn // IEEE Trans. Commun. - Aug. 2002. - Vol. 50, No. 8. - P. 1293-1300. - DOI : 10.1109/TCOMM.2002.800829.

3. Andrews L. C. Laser Beam Propagation through Random Media / Larry C. Andrews, Ronald L. Phillips. SPIE Press, 1998. — ISBN : 9780819459480. 
4. Majumdar A. K. Advanced Free Space Optics (FSO): A Systems Approach / Arun K. Majumdar. - New York : Springer-Verlag, $2015 . \quad-\quad$ ISBN : 9781493909179. — DOI : 10.1007/978-1-4939-0918-6.

5. Churnside J. H. Aperture averaging of optical scintillations in the turbulent atmosphere / James $\mathrm{H}$. Churnside // Appl. Optics. - 1991. — Vol. 30, No. 15. P. 1982-1994. - DOI : 10.1364/AO.30.001982.

6. Aperture-averaging effects for weak scintillations / Gerald E. Homstad, John W. Strohbehn, Roger H. Berger, J. M. Heneghan // JOSA A. - 1974. - Vol. 64, No. 2. - P. 162-165. — DOI : 10.1364/JOSA.64.000162.

7. Navidpour S. M. BER performance of free-space optical transmission with spatial diversity / S. Mohammad Navidpour, Mural Uysal, Mohsen Kavehrad // IEEE Trans. Wireless Commun. - Aug. 2007. - Vol. 6, No. 8. - P. 2813-2819. — DOI : 10.1109/TWC.2007.06109.

8. Shannon capacities and error-correction codes for optical atmospheric turbulent channels / Jaime A. Anguita, Ivan B. Djordjevic, Mark A. Neifeld, Bane V. Vasic // J. Optical Networking. - 2005. - Vol. 4, No. 9. - P. 586-601. - DOI: 10.1364/JON.4.000586.

9. Average capacity of optical wireless communication systems over atmospheric turbulence channels / Hector E. Nistazakis, Evangelia A. Karagianni, Andreas D. Tsigopoulos, Michael E. Fafalios, George S. Tombras // J. Lightwave Technol. - Apr. 2009. - Vol. 27, No. 8. - P. 974-979. - DOI : 10.1109/JLT.2008.2005039.

10. Haas S. M. Capacity of wireless optical communications / S. M. Haas, J. H. Shapiro // IEEE J. Sel. Areas Commun. - Oct. 2003. - Vol. 21, No. 8. - P. 1346-1357. — DOI : 10.1109/JSAC.2003.816618.

11. Fading reduction by aperture averaging and spatial diversity in optical wireless systems / Mohammad-Ali Khalighi, Noah Schwartz, Naziha Aitamer, Salah Bourennane // J. Optical Commun. Networking. - 2009. - Vol. 1, No. 6. - P. 580-593. - DOI : 10.1364/JOCN.1.000580.

12. Lee E. J. Part 1: Optical communication over the clear turbulent atmospheric channel using diversity / E. J. Lee, V. W. S. Chan // J. Sel. Areas Commun. - Nov.
2004. - Vol. 22, No. 9. - P. 1896-1906. - DOI : 10.1109/JSAC.2004.835751.

13. Ibrahim M. M. Performance analysis of optical receivers with space diversity reception / M. M. Ibrahim, A. M. Ibrahim // IEE Proc. Commun. - Dec. 1996. Vol. 143, No. 6. - P. 369-372. - DOI : 10.1049/ip-com:19960885.

14. Diversity techniques for a free-space optical communication system in correlated log-normal channels / Mohamed Abaza, Raed Mesleh, Ali Mansour, El-Hadi M. Aggoune // Opt. Eng. - Jan. 2014. - Vol. 53, No. 1. - P. 016102. — DOI : 10.1117/1.OE.53.1.016102.

15. Free-space optical communication employing subcarrier modulation and spatial diversity in atmospheric turbulence channel / W. O. Popoola, Z. Ghassemlooy, J. I. H. Allen, E. Leitgeb, S. Gao // IET Optoelectronics. Feb. 2008. - Vol. 2, No. 1. - P. 16-23. - DOI : 10.1049/iet-opt:20070030.

16. Goldsmith A. Wireless Communication / Andrea Goldsmith. - Cambridge University Press, 2005. ISBN : 9780521837163.

17. Rosenblueth E. Point estimates for probability moments / Emilio Rosenblueth // Proc. NAS. - 1975. Vol. 72, No. 10. - P. 3812-3814. - DOI : 10.1073/pnas.72.10.3812.

18. Karmeshu. On the applicability of average channel capacity in log-normal fading environment / Karmeshu, Vineet Khandelwal // Wireless Personal Commun. - Feb. 2013. - Vol. 68, No. 4. - P. 1393-1402. - DOI : 10.1007/s11277-012-0529-2.

19. Holtzman J. M. A simple, accurate method to calculate spread-spectrum multiple-access error probabilities / J. M. Holtzman // IEEE Trans. Commun. Mar. 1992. - Vol. 40, No. 3. - P. 461-464. - DOI : $10.1109 / 26.135712$.

20. Laourine A. Estimating the ergodic capacity of log-normal channels / A. Laourine, A. Stephenne, S. Affes // IEEE Commun. Lett. — Jul. 2007. — Vol. 11, No. 7. P. 568-570. - DOI : 10.1109/LCOMM.2007.070302.

21. Optical Wireless Products. - URL : http://www.fsona.com/products.php. 